\title{
PLANEAMIENTO URBANÍSTICO Y FORMA URBANA EN CATALUÑA. EL CASO DE LA REGIÓN DE GIRONA $(1979-2006)$
}

\author{
Juli Valdunciel Coll \\ Departamento de Geografía. Universitat de Girona \\ juli.valdunciel@udg.edu
}

\section{RESUMEN}

La dinámica de urbanización y los cambios en el paisaje urbano en Cataluña y España han sido enormes en los últimos años. Este artículo lo ejemplifica a partir del caso de la región de Girona (1979-2006). Los resultados permiten validar la consolidación del fenómeno de la urbanización dispersa y la aparición de un paisaje de expansión formado por tres tipologías morfológicas: ensanches, urbanizaciones y polígonos de actividad económica. Esto pone de manifiesto las carencias del planeamiento para ordenar el desarrollo urbano y una degradación del modelo territorial mediterráneo.

Palabras clave: globalización, planeamiento, urbanización dispersa, posmodernidad.

\section{ABSTRACT}

Urbanisation and transformations of the urban landscape in Catalonia and Spain were enormous in the recent years. This article demonstrates this through the case study of Girona region (1979-2006). The results point to the consolidation of the dispersed city phenomenon and the emergence of a new landscape comprising three morphological types: urban extensions, low density residential estates and industrial zones. This reveals shortcomings of planning for urban growth and a degradation of the Mediterranean territorial model.

Key words: globalisation, spatial planning, urban sprawl, post modernism.

Fecha de recepción: mayo 2012.

Fecha de aceptación: julio 2013. 


\section{INTRODUCCIÓN}

El crecimiento urbano ha sido un rasgo distintivo del modelo económico español en su transición hacia la globalización. La entrada en la Unión Europea (UE) y la recuperación económica marcaron, a partir de 1986, el inicio de un nuevo ciclo de expansión basado en el desarrollo de los servicios y el sector inmobiliario. Después de un breve período de recesión (1992-1995), en 1996 la economía remprendió de nuevo y culminó con un crecimiento sin precedentes. Las causas de la denominada burbuja inmobiliaria fueron la especialización en el turismo residencial y la liberalización de la economía, pero también lo fueron variables sociales, como la reducción de personas por hogar, el crecimiento demográfico y la consideración de la vivienda como un bien de inversión. Estos factores reforzaron la oferta, formada por una coalición entre grandes empresas inmobiliarias y el sector financiero, e impulsaron una demanda aparentemente solvente que, a pesar de los sucesivos aumentos en el precio de la vivienda, pudo concurrir en el mercado gracias a la facilidad de acceso al crédito hipotecario. La crisis global iniciada a finales de 2007 puso fin a este ciclo y abrió un nuevo periodo de incertidumbre en el cuál todavía hoy estamos inmersos (Romero, 2010; Naredo y Montiel, 2011; Romero, 2011).

La política urbanística facilitó ésta dinámica de manera poco responsable. Por una parte, las administraciones locales emprendieron una competición para proveer suelo, atraer inversiones y aumentar así los recursos de las arcas municipales. Por otra parte, las administraciones autonómicas no legislaron o desplegaron a tiempo los instrumentos de planeamiento territorial (Burriel, 2008 y 2011). En el caso catalán, la aprobación por parte de la Generalitat del Plan Territorial General de Catalunya en 1995 quedó como un ejercicio aislado. No fue hasta el 2002 cuando se introdujo el principio de sostenibilidad en el planeamiento a través de la nueva Ley de Urbanismo catalana y hasta 2004 cuando el gobierno tripartito empezó a implementar, a través de su Programa de Planeamiento Territorial, planes territoriales y urbanísticos supramunicipales para el conjunto del territorio (Plan Director Urbanístico del Sistema Costero de Cataluña, 2005; Plan Territorial de l'Empordà, 2006; etc.) (Nel·lo, 2010). El resultado fue, dadas las nuevas condiciones tecnológicas, los diferenciales en la renta del suelo y el hecho que el planeamiento urbanístico es suficiente para la cobertura técnica y jurídica de cualquier actuación, una explosión de la urbanización sin una solución de coordinación territorial (Esteban, 2003).

Así pues, la urbanización dispersa como fenómeno de dispersión, especialización funcional y separación de los grupos sociales que caracteriza la ciudad del capitalismo avanzado (Indovina, 1990; Monclús, 1998; Nel·lo, 2001) se manifestó también en el caso español, aunque tamizada por algunas variables locales relacionadas con el modelo socioeconómico y el planeamiento. En este sentido, el paisaje urbano heredado reflejaría las características de la nueva ciudad postmoderna (mercantilización, privatización y tematización), tal y como han descrito diversos autores (Ellin, 1999; Amendola, 2000; Gospodini, 2006), pero según una versión muy marcada, especialmente en la costa Mediterránea, por las morfologías turístico-residenciales (VVAA, 2006). Finalmente, este proceso habría comportado un gran impacto territorial, con costes derivados del consumo masivo de suelo, la fragmentación social del espacio y la banalización del paisaje (Rueda, 2002; Muñoz, 2004; Muñiz, Calatayud y Garcia, 2007; Nogué, 2007). Por todo ello, la comprensión de esta etapa pone numerosos interrogantes no sólo sobre la magnitud de la urbanización sino también sobre el papel jugado por el planeamiento y los agentes, y la calidad del modelo territorial resultante. 
Este artículo sintetiza los resultados de una tesis doctoral que ha tenido como objeto hacer un balance del proceso de urbanización en la región de Girona desde la instauración de los ayuntamientos democráticos, en 1979, hasta 2006, justo antes de la crisis actual (Valdunciel, 2011). La metodología se ha basado en el análisis de los planes parciales de urbanización aprobados, es decir, los documentos que han permitido nuevos desarrollos en suelo urbanizable. Posteriormente, se ha contrastado la ejecución de dichos planes, a través del ortofotomapa en-linea del Instituto Cartográfico de Cataluña (ICC), para precisar sus efectos territoriales reales. La interpretación de los resultados se ha hecho a partir de dos perspectivas complementarias: la regional, con el objetivo de comprender la producción del espacio de acuerdo con las lógicas de urbanización difusa, y la del lugar, con el objetivo de identificar las principales tipologías morfológicas. Se trata, tanto por la dimensión temporal contemplada (1979-2006) como por la magnitud de las fuentes manejadas (522 planes parciales) de un estudio sin precedentes para la comprensión de la urbanización en España durante el periodo Democrático. Esto ha sido en buena parte posible gracias al hecho que a partir de 2007, y por primera vez, el planeamiento vigente ha estado disponible en internet a través de Registro de Planeamiento Urbanístico de Cataluña (RPUC).

\section{EL ESCENARIO DEL PLANEAMIENTO}

La región de Girona es una de las siete áreas de planificación de la Generalitat de Cataluña. Se trata del ámbito de la provincia homónima, a excepción de la comarca de la Cerdanya (ver figura 1). Cuenta con 211 municipios y una población de 700.000 habitantes organizados alrededor de Girona, un área urbana de 150.000 habitantes que es el nexo de un sistema de ocho pequeñas áreas urbanas (Figueres, Olot, Banyoles, etc.) y de la Costa Brava, un ámbito turístico de proyección internacional (Castañer, Gutiérrez y Vicente, 2009) (ver figura 2). A su vez, dicho conjunto está estrechamente imbricado en la región metropolitana de Barcelona (RMB) y forma parte del eje transeuropeo Mediterráneo (ver Vicente, 2003).

Entre finales de 1978 y 1979 la Generalitat asumió las competencias en materia de urbanismo y ordenación del territorio, y se instauraron los primeros ayuntamientos democráticos. En un contexto de gran confianza en las posibilidades de los nuevos poderes públicos, los principales municipios de la región de Girona iniciaron la revisión de su planeamiento general y a lo largo los años siguientes se redactaron planes nuevos en los municipios más pequeños. Los planes elaborados durante los primeros años de la Democracia proyectaron los escenarios de desarrollo urbano para los siguientes 15-20 años.

Los primeros planes democráticos tenían como objetivo moderar el crecimiento, suturar la trama urbana y resolver las carencias heredadas del desarrollismo franquista (Ferrer y Sabaté, 1999). En este contexto, el plan parcial emergió como una herramienta estratégica para definir la morfología urbana y conseguir infraestructuras, espacios públicos y equipamientos. Pero en la práctica aparecieron dos órdenes de problemas. En primer lugar, los redactores tuvieron que lidiar con la presencia de importantes bolsas de suelo urbanizable clasificado (en algunos casos con plan parcial aprobado) y numerosas urbanizaciones ilegales. Como consecuencia, y para evitar la conflictividad con la propiedad y con los colectivos de parcelarios, la solución pasó por la incorporación de éstos grandes volúmenes de suelo urbanizable en los nuevos planes de urbanismo (este fue el caso de los planes de Lloret de Mar, 706 ha; Vidreres, 613 ha; 
Figura 1

LA REGIÓN DE GIRONA EN EL CONTEXTO DE CATALUÑA

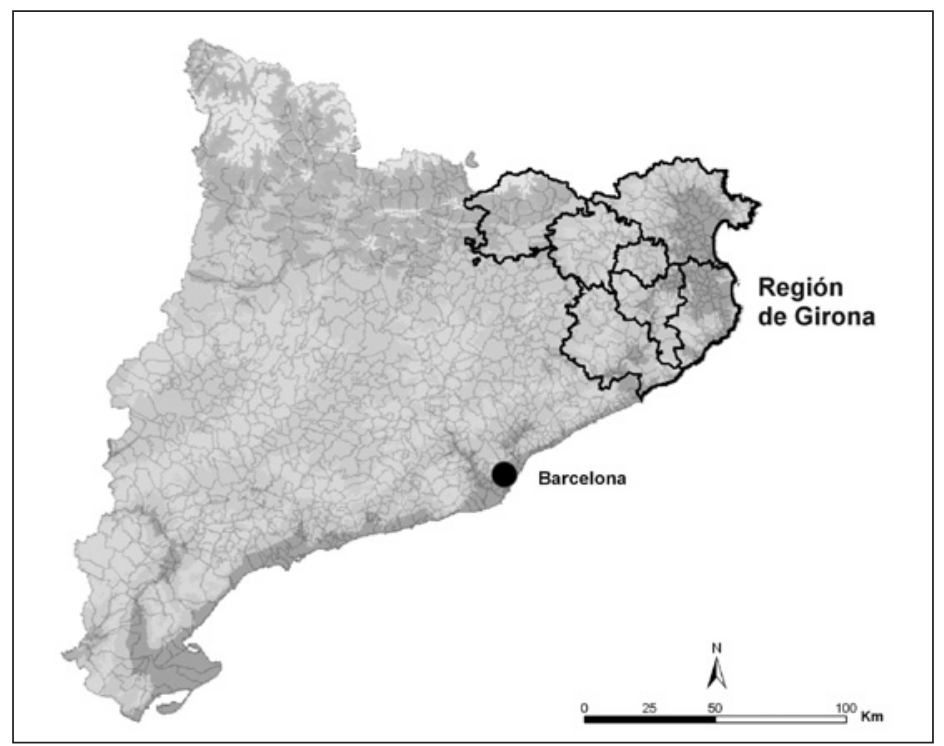

Fuente: Elaboración propia.

Figura 2

LA REGIÓN DE GIRONA, PRINCIPALES ÁREAS URBANAS

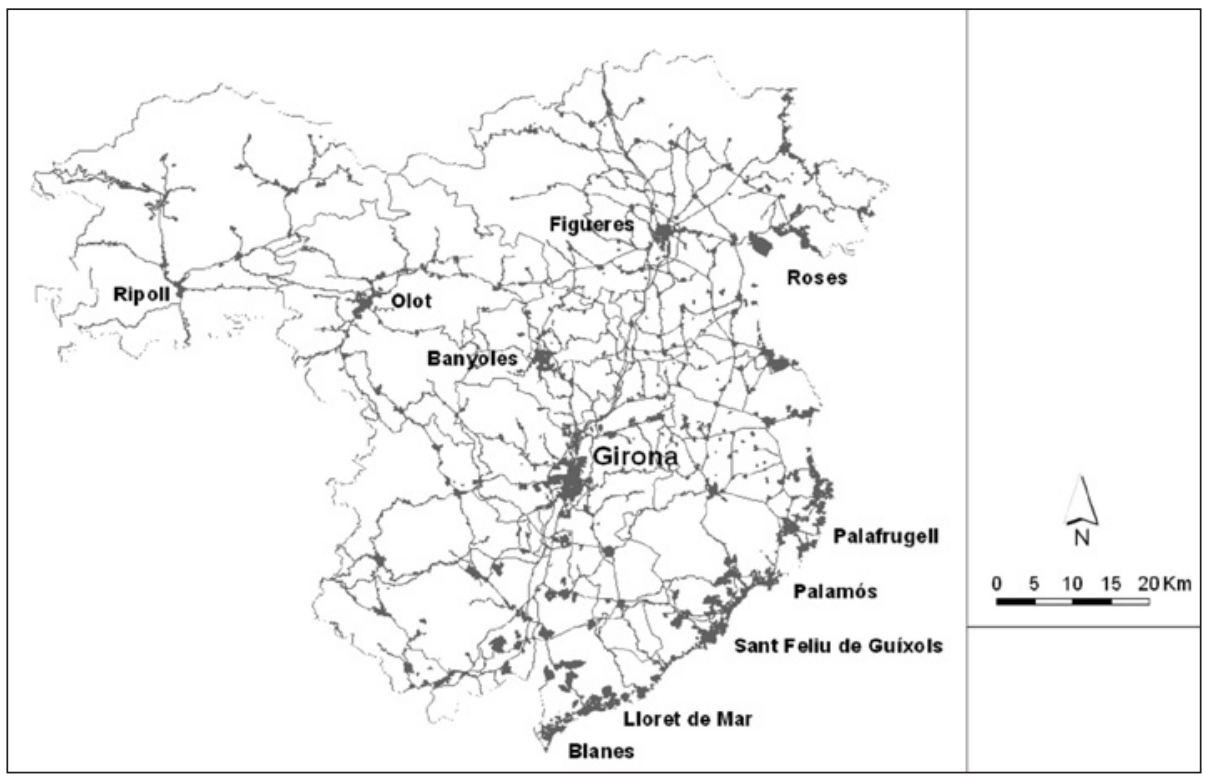

Fuente: Elaboración propia. 
Celrà, 346 ha). En segundo lugar, los planes supramunicipales aprobados para las áreas urbanas (Girona y Figueres son los ejemplos más destacados) fueron inmediatamente desmantelados después de las elecciones de 1980, en la medida que el nuevo gobierno de Convergència i Unió entendió que la ordenación urbana era una tarea municipal (Vicente, 1998; Esteban, 2003). Como consecuencia, los municipios empezaron a redactar o a revisar los planes según sus propias expectativas y muchos clasificaron el suelo con criterios sobredimensionados. En el área urbana de Girona y en la costa, por ejemplo, un tercio de los planes de urbanismo vigentes en el año 2000 (la mayoría redactados durante la década de 1980 y la primera mitad de 1990) clasificaban el suelo urbanizable en una medida igual o superior al preexistente.

Así pues, el escenario del planeamiento estuvo marcado por dos tipos de paradojas. Por una parte, la inercia del desarrollismo, hecho que convirtió a muchos de los nuevos planes de urbanismo, de facto, en proyectos de expansión. Por otra parte, la ausencia de escenarios de planificación adecuados a la escala de los fenómenos de orden territorial. De esta manera, junto a los planes responsables, orientados a la mejora morfológica y a la dotación de servicios, aparecieron nuevos planes diseñados para competir por los efectos de la difusión urbana, principalmente en los municipios pequeños.

\section{LA PRODUCCIÓN DEL ESPACIO EN EL ÁMBITO REGIONAL}

Entre 1979 y 2006 se aprobaron en la región de Girona 522 planes parciales de urbanización, con una superficie de 5.930 ha. El 69\% de la superficie urbanizada ocupó suelo virgen mientras que el $31 \%$ restante correspondió a suelos débilmente ocupados por antiguas operaciones ilegales, subsiguientemente consolidadas (ver tabla 1). Por usos, las principales categorías de plan fueron el residencial (57\%), seguido del industrial (27\%) y el mixto (principalmente residencia y servicios) (12\%).

Figura 3

PLANES PARCIALES APROBADOS, EVOLUCIÓN TEMPORAL, 1979-2006

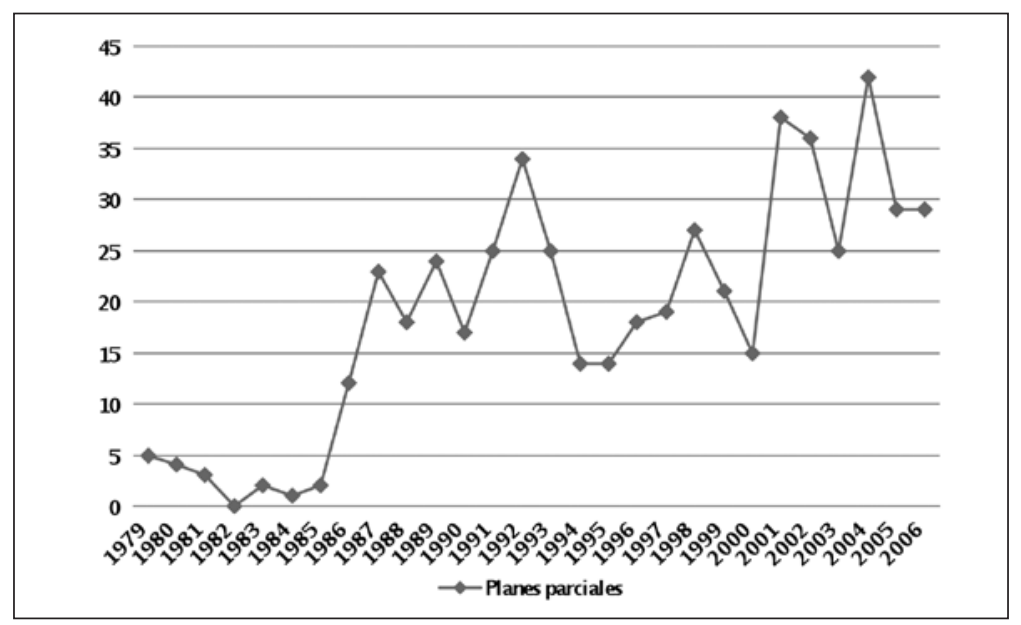

Fuente: Elaboración propia a partir del Registro de Planeamiento Urbanístico de Cataluña. 
En el plano temporal se pueden distinguir cuatro fases (ver figura 3). La primera comprende el periodo que va de 1979 a 1985, fue de débil crecimiento y está vinculada a los efectos de la crisis de la década de 1970. La segunda va de 1986 a 1992 y estuvo marcada por la entrada de España en la UE y la recuperación de la economía, factores que impulsaron la llegada de inversiones y el inicio de un ciclo de expansión, con un pico de más de 30 planes en 1992. La tercera va de 1993 a 1995 y estuvo marcada por un breve periodo de recesión, que se saldó con la devaluación de la peseta, la introducción de medidas de flexibilización en el mercado laboral y la liberalización de la economía. Una cuarta fase de crecimiento se inició a partir de 1996 y ésta se extendió, con algunas oscilaciones, hasta 2006. Este último ciclo de expansión fue incluso superior al primero, tanto en términos de planes aprobados (el $54 \%$ ) como de su duración, más de 10 años.

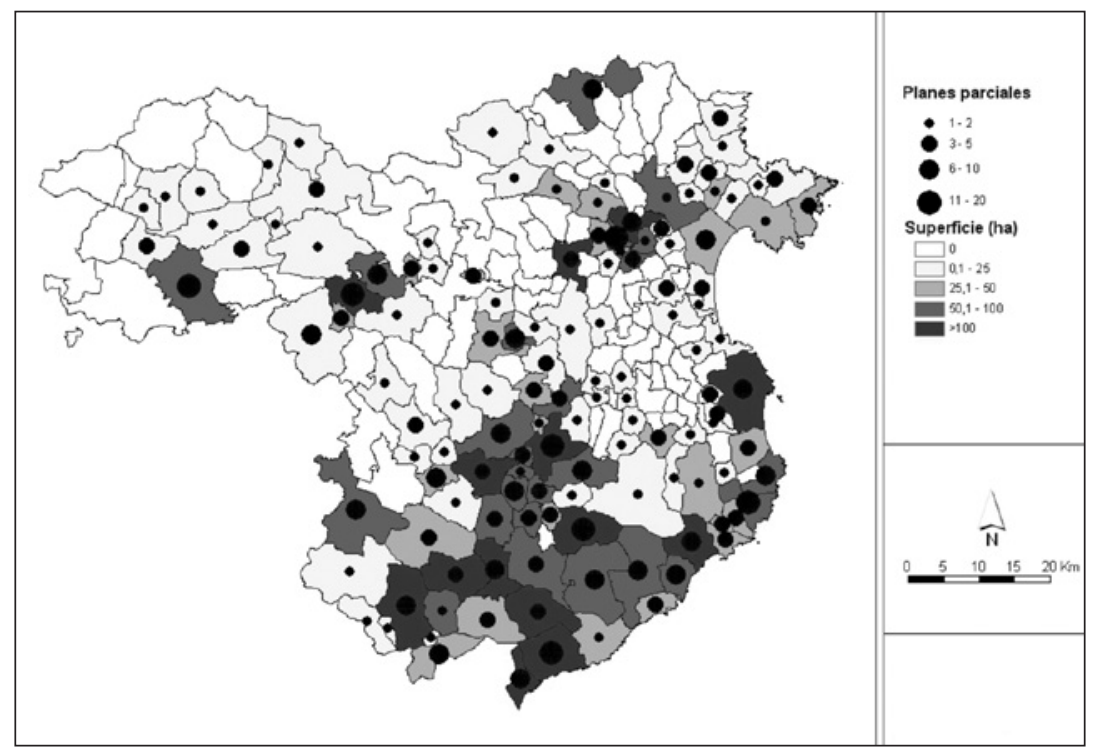

Fuente: Elaboración propia a partir del Registro de Planeamiento Urbanístico de Cataluña.

Por lo que se refiere a la distribución espacial se pueden identificar tres áreas diferenciadas (ver figura 4). En primer lugar la costa, que experimentó un gran crecimiento vinculado a la transición del turismo tradicional (modelo fordista) al residencialismo o turismo de residencia en propiedad (ver Fraguell, 1994; Donaire, 1996). Además, y por primera vez, ésta dinámica afectó también a los municipios próximos de segunda línea de costa, especialmente en el Ampurdán, el área patrimonial y ambientalmente más cualificada. En segundo lugar, las áreas urbanas, donde el desarrollo afectó no sólo a los núcleos principales sino, especialmente, a los municipios de sus coronas adyacentes (en los casos de Girona, Figueres y Olot, un $76 \%, 84 \%$ y $67 \%$ de los planes correspondieron, respectivamente, a los municipios de su entorno). En tercer lugar a los que se ha optado por denominar «territorios en red», es decir, 
los municipios situados sobre los corredores viarios que unen Girona con el resto de ciudades, la costa y la RMB. Se trata de zonas de urbanización difusa de carácter lineal y de núcleos ubicados en sus puntos de intersección que han crecido siguiendo una suerte de inversión de los lugares centrales tradicionales (Maçanet de la Selva, Llagostera, La Jonquera).

Por lo que hace referencia a la dispersión, el estudio ha utilizado una noción amplia del concepto que incluye no sólo la ausencia de continuidad física (el fenómeno más evidente) sino también la ausencia de proporcionalidad entre un plan y el núcleo urbano preexistente, es decir, la presencia de planes fuera de escala. Como resultado se pone de manifiesto que el crecimiento compacto fue mayoritario, con un 56\% de los planes, pero el crecimiento disperso representó, con un $61 \%$ de la superficie, la mayor parte del suelo ordenado. Si bien hay que matizar que de dicho $61 \%$ la mitad correspondió a la regularización de las citadas operaciones ilegales preexistentes, mayormente urbanizaciones de baja densidad muy extensivas, no es menos cierto que la otra mitad fueron, desgraciadamente, planes de nueva planta incoherentes desde el punto de vista de la ordenación territorial (ver tabla 1).

Tabla 1

SÍNTESIS DE LAS PRINCIPALES MAGNITUDES, 1979-2006

\section{Datos generales}

1. Total planes parciales

\section{Génesis de los planes}

- Nueva planta

- Regularización actuaciones ilegales

\section{Tipo de desarrollo}

- Compacto

- Disperso / fuera de escala
Planes parciales Superfície (ha)

522

5.930

$453(87 \%)$

$4.095(69 \%)$

$68(13 \%)$

$1.832(31 \%)$

Fuente: Elaboración propia.

Este último problema afectó particularmente los municipios pequeños de la costa, la segunda línea de costa y los entornos de las áreas urbanas, y estuvo estrechamente asociado a la presencia de planes de urbanismo sobredimensionados o bien a su modificación «a la carta», ya fuera en el momento de su redacción o a través de modificaciones puntuales. Este último fue el caso de diversas urbanizaciones y espacios industriales (se han documentado 6 casos de urbanizaciones y 13 de espacios industriales, pero se sospecha que pudieron ser muchos más) y el modus operandi fue siempre similar. Los promotores urbanos desplazaron su campo de acción hacia el traspaís, donde encontraron no sólo suelo rústico más barato sino también unas administraciones municipales más proclives para recalificarlo. Una vez aprobados inicialmente los planes parciales, promotores, ayuntamientos e incluso organizaciones sociales, como los sindicatos, hicieron de palanca sobre la comisión provincial de urbanismo para promover su aprobación. La justificación utilizada fue generalmente la falta de suelo o bien la necesidad de promover una cierta visión del «desarrollo local» (creación de empleo, mejora de la arcas 
municipales, etc.). El precio fue la apropiación privada de elevadas plusvalías territoriales. Tan sólo la amenaza de urbanizar algunos de los últimos parajes vírgenes del litoral activó conflictos iniciados por los grupos ecologistas, algunos de los cuáles se saldaron con la paralización de los planes previstos (este fue el caso de los proyectos de Castell, Pinya de Rosa y Cap Ras) (ver Nel·lo, 2003 y Territori - Observatori de projectes i debats territorials de Catalunya, 2012).

Finalmente, por lo que se refiere a los promotores hay que señalar dos aspectos. Por una parte, y en el plano temporal, se aprecia la transición de una situación próxima al equilibrio entre promoción privada y pública (etapa 1979-1996) a una situación de dominio de la promoción privada que coincide con el giro liberalizador en las políticas públicas y el ciclo más intenso de crecimiento (etapa 1997-2006). Por otra parte, la relación entre promoción y territorio permite definir tres grandes ámbitos de mercado. En primer lugar la costa, con un predominio de los agentes privados, un grado de apertura al capital exterior destacado (región metropolitana de Barcelona e internacional) y una especialización en el segmento residencial-turístico. En segundo lugar, las áreas urbanas de Girona y Figueres, caracterizadas por el liderazgo de los agentes locales, un grado de contención elevado y un producto inmobiliario diversificado (residencial e industrial). En tercer lugar, y último, las comarcas interiores, con un predominio de los agentes públicos (ayuntamientos y Generalitat, a través del Institut Català del Sòl), un grado de contención elevado y un producto inmobiliario industrial o residencial de contenido social. En todo caso, la atomización de la promoción fue un hecho. Así que, a parte de los grandes promotores de Barcelona asociados a los nichos de mercado más provechosos ( $9 \%$ de los planes) y el Institut Català del Sòl (6\%) (el promotor público más destacado), los promotores locales (una miríada de agentes que incluyó a pequeñas promotoras, «industriales» convertidos en promotores y personas físicas asociadas a proyectos de oportunidad) fueron los agentes más destacados.

\section{LAS TIPOLOGÍAS URBANÍSTICAS}

El análisis de los planes parciales y su comparación permite afirmar que el modelo de extensión urbano se desarrolló a partir de tres grandes tipologías urbanísticas: (1) ensanches, (2) urbanizaciones y (3) polígonos de actividad económica (ver tabla 2). Éstas representaron, en sus diferentes variantes, el $98 \%$ de los planes analizados. Tan sólo un 2\% correspondió a otras fórmulas singulares, como los polígonos de equipamientos o las zonas hoteleras.

La primera tipología en cuanto a número de actuaciones fueron los ensanches, con 236 planes parciales ( $46 \%$ del total y $26 \%$ de la superficie). Se trata de crecimientos residenciales compactos y con densidades medias y altas (la media fue 34 viviendas/ha). Su desarrollo abarcó el conjunto del territorio, aunque hubo una diferencia destacable entre las ciudades, donde predominaron los proyectos de densidad media y alta (ver figura 5), de los municipios pequeños, donde lo hicieron las soluciones de densidad media y baja. La idea de ensanche como trazado regular, dividido en manzanas con la edificación plurifamiliar, se remonta a las ideas de Ildefonso Cerdà y su ensanche para Barcelona de la segunda mitad del siglo XIX. El modelo de ensanche prosiguió su desarrollo durante la primera mitad del siglo XX, fue matizado por la Ley del suelo de 1956 y su reforma de 1975 y, con la instauración de la Democracia, se convirtió la fórmula por excelencia para modelar la morfología urbana y resolver los déficits heredados del régimen anterior. 
Tabla 2

TIPOLOGÍAS URBANÍSTICAS, 1979-2006

\begin{tabular}{lcc}
\hline Tipologías & Planes parciales & Superfície (ha) \\
\hline 1. Ensanches & $236(46 \%)$ & $1.505(26 \%)$ \\
2. Polígonos de actividad económica & $171(33 \%)$ & $2.036(34 \%)$ \\
3. Urbanizaciones & $103(19 \%)$ & $2.315(39 \%)$ \\
4. Otras & $12(2 \%)$ & $74(1 \%)$ \\
\hline
\end{tabular}

Fuente: Elaboración propia.

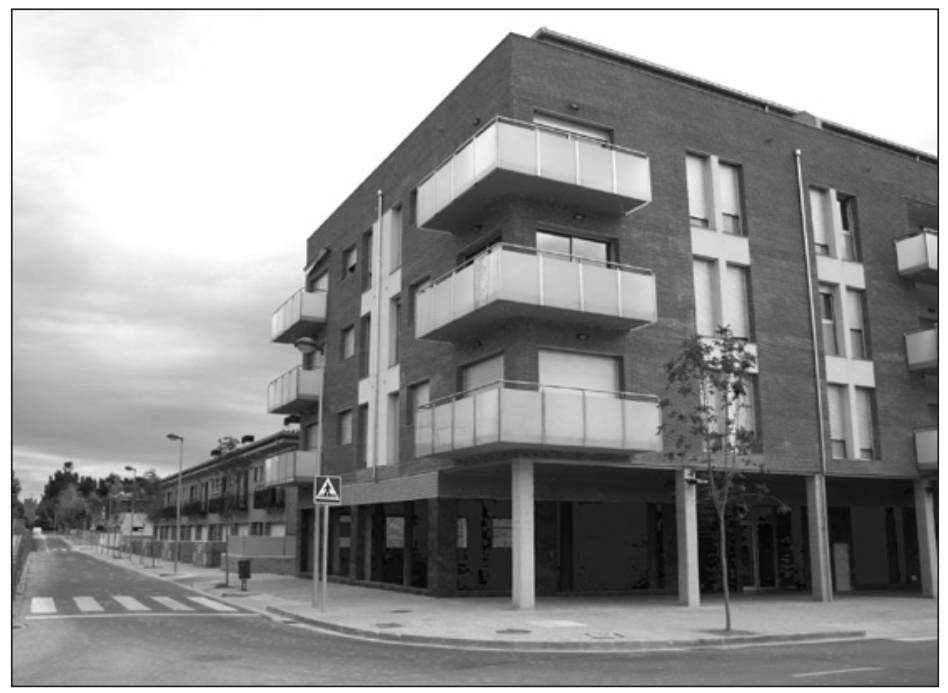

Fuente: Elaboración propia.

Desde una perspectiva social, ciertamente las soluciones de ensanche con densidades razonablemente elevadas han hecho posible la creación de nuevos ambientes urbanos con actividades comerciales y espacios públicos (plazas, ramblas y equipamientos) que han incidido en el fomento de las relaciones de comunidad. Desde una perspectiva ambiental, esto ha significado crecer con consumos de suelo por habitante ajustados y la creación de tramas urbanas compactas que han facilitado los desplazamientos de proximidad. Así pues, por sus características (compacidad, densidad y mezcla de usos y de grupos sociales) los ensanches desarrollados representaron, por lo general, una continuidad con el modelo de ciudad mediterránea. Esto no es óbice para dejar de señalar una tendencia progresiva al reforzamiento de la autonomía formal de los proyectos y a la creación de ambientes privativos (soluciones 
edificatorias singularizantes, ubicación introspectiva de los espacios públicos, etc.) que ha ido asentando una cierta división entre vida comunitaria y vida urbana. Este proceso es especialmente acuciante en los municipios de pequeña dimensión de la periferia de las ciudades, donde a partir de la década de 1990 proliferaron actuaciones con vivienda unifamiliar a medio camino entre la idea de ensanche y la de urbanización de baja densidad.

Todavía en la esfera del espacio residencial, una segunda tipología fueron las urbanizaciones, con 103 planes parciales (19\% del total y $39 \%$ de la superficie). Se trata de desarrollos residenciales dispersos y de baja densidad, generalmente de tipo ciudad-jardín y de segunda residencia (la media fue 14 viviendas/ha). En el mundo Mediterráneo dicho modelo tiene una raíz histórica dual. Por una parte, es cierto que este deriva, como se apunta generalmente, de las influencias recientes del modelo suburbano anglosajón. Por otra parte, no es menos cierto que la residencia de baja densidad tiene una historia de al menos un siglo en Cataluña (Franquesa, 2010), y que la vivienda unifamiliar ha sido el modelo dominante históricamente en el mundo rural. En cualquier caso, no fue hasta la década de 1960 cuando la socialización del veraneo y la llegada del turismo europeo abonaron la proliferación en masa urbanizaciones de tipo ciudad-jardín, principalmente en el litoral. Así, muchos promotores radicados generalmente en Barcelona vieron un negocio provechoso en este tipo de actuaciones, aunque fuera a costa de urbanizar de manera ilegal y de vender parcelas sin los servicios básicos (asfaltado, luz y agua). Como consecuencia, con la llegada de la Democracia la administración se vio obligada a legalizar y dotar de infraestructuras a muchas urbanizaciones, un proceso que, dada la reducida capacidad financiera de los ayuntamientos y de los parcelistas, hizo necesario recurrir a su consideración como suelo urbanizable y a su compleción, e incluso ampliación, a través de un nuevo plan parcial (ver figura 6).

A partir de la segunda mitad de la década de 1980 la demanda de segunda residencia repuntó de nuevo, ahora bajo un nuevo tipo de proyecto de perfil posmoderno (urbanizaciones asociadas a equipamientos de ocio y urbanizaciones de muy baja densidad) presentado como una alternativa de mayor calidad al modelo de «sol y playa» tradicional. Este tipo de actuaciones presentan los valores de maximización de la privacidad de las urbanizaciones preexistentes pero a través de una versión más sofisticada que incorpora, ya sea una visión idealizada del paisaje local, generalmente del Ampurdán, sus mitos y su patrimonio (Dalí, los núcleos medievales y el paisaje agrario) o bien un referente temático reconocido globalmente, como es un campo de golf. Concretamente, durante el perído de estudio se aprobaron 6 campos de golf asociados a desarrollos urbanísticos. Algunos proyectos, como Residencial Torre Mirona golf club (Navata), incorporan incluso dispositivos de defensa (vallas exteriores, vigilancia privada), hecho que muestra una influencia creciente del modelo de las gated communities norteamericanas (ver Bellet, 2007). Se trata de planes que cumplen el estándar técnico definido por la ley, pero con unos criterios de localización y unas ratios de consumo de suelo absolutamente insostenibles. Este es el caso, por ejemplo, del citado plan Residencial Torre Mirona golf club (Navata), que con 122 ha triplicó las 33 ha de suelo urbano del municipio, y del Sector UR-3 Golf (Peralada), que con 70 ha duplicó las 35 ha de suelo urbano del municipio. No en vano, las urbanizaciones fueron, con 2.315 ha, la primera tipología en términos de superficie ocupada durante el periodo estudiado. 


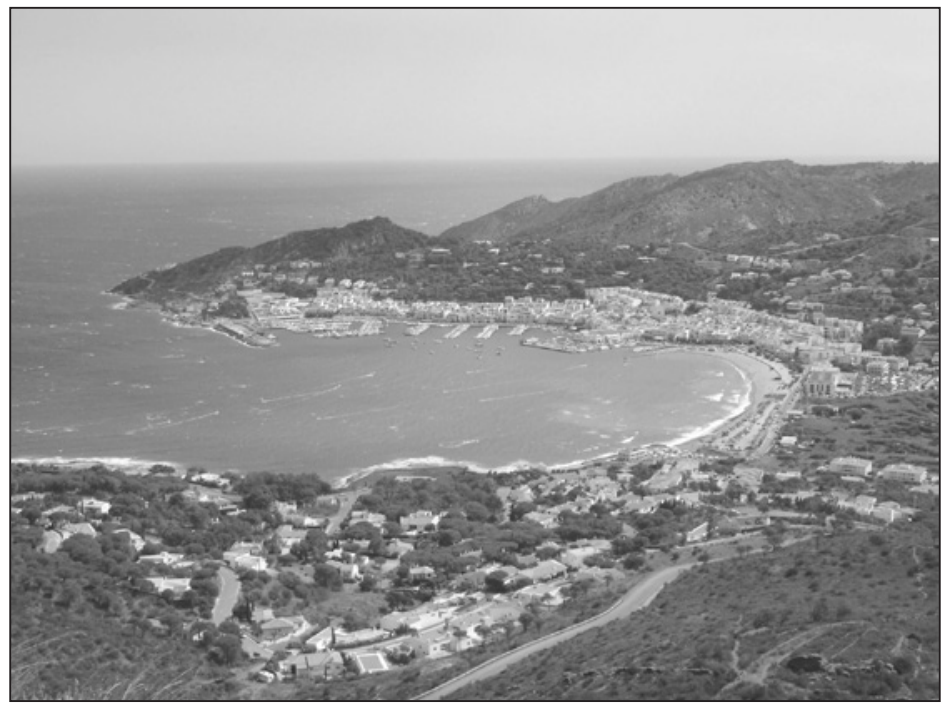

Fuente: Elaboración propia.

Pero además, y tal y como se ha apuntado anteriormente, en los municipios de pequeña dimensión de la periferia de las ciudades proliferaron, durante la década de 1990, conjuntos residenciales de baja densidad de primera residencia. Se asistió en estas áreas a una creciente hibridación entre la idea de ensanche de primera residencia y la de urbanización de segunda residencia (la vivienda unifamiliar es el referente común), cada vez más difícil de diferenciar. Se trata de de los característicos paisajes «urbanales» que rodean las ciudades descritos por Muñoz (2004) en el área de Barcelona. En los casos en que se trataba de operaciones compactas, con una densidad media superior a las 20 viviendas/ ha y ajustadas a la escala del núcleo preexistente, se las ha considerado ensanches de baja densidad. En el resto de casos, caracterizados por su localización dispersa o, aún teniendo una ubicación compacta, presentaban una densidad inferior al umbral citado (se trata de un umbral empírico por debajo del cuál se ha considerado que no se genera un tejido urbano mínimamente compacto), se las ha incluido en el grupo de las urbanizaciones, con sus problemáticas y costes asociados (ver figura 7). A modo de conclusión, hay que señalar que la densidad media de las actuaciones residenciales descendió de 30 a 24 viviendas/ha entre el primer y el último quinquenio del periodo estudiado, dato que corrobora la transición hacia una cultura del habitar de (más) baja densidad, a la que contribuyó, de manera creciente, la primera residencia.

La tercera tipología fueron los polígonos de actividad económica, con 171 planes parciales (33\% del total y $34 \%$ de la superficie). Se trata de actuaciones destinadas a actividades de producción, almacenamiento y servicios, generalmente separadas del resto de la trama urbana. Hasta la década de 1970 las empresas se instalaban de manera espontánea y presentaban graves carencias de infraestructuras y servicios. Con la llegada de la Democracia la Generalitat impulsó, a través del Institut Català del Sòl, la promoción de suelo a gran escala 


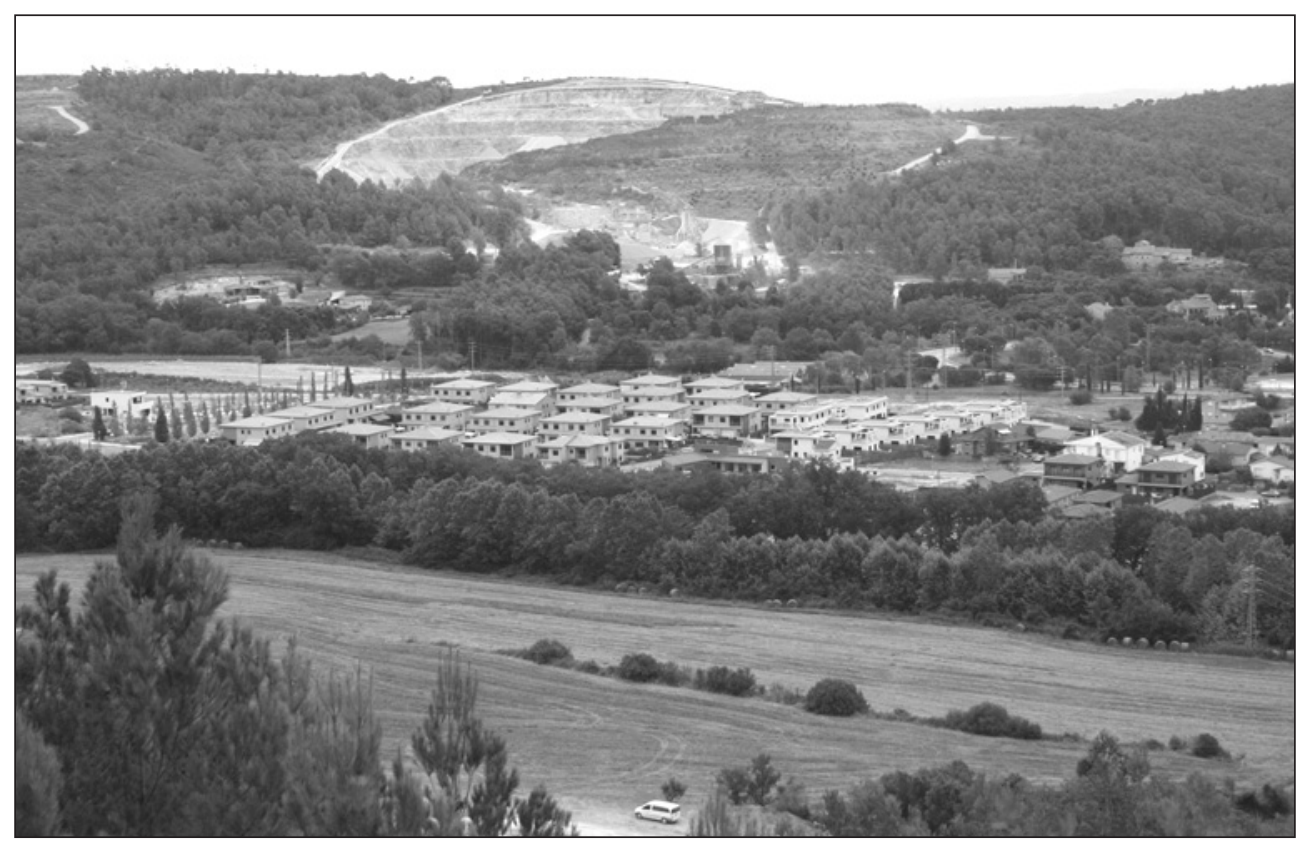

Fuente: Elaboración propia.

para actividades económicas, especialmente en los municipios pequeños de la periferia de las ciudades. La creación de actuaciones con un gran valor estratégico y ubicaciones centrales en el marco de las áreas urbanas alternó con proyectos dispersos, sujetos, en muchos casos, a la discrecionalidad de factores políticos locales, o, simplemente, a la competencia entre municipios por fijar inversiones en un marco de ausencia de planeamiento territorial.

La terciarización y globalización de la economía de la década de 1990 marcaron la transición de la idea de polígono industrial, compuesto por fábricas y talleres, a la de polígono de actividad económica, donde las industrias conviven con las oficinas y el comercio. Como consecuencia, nuevas formulas comerciales (centros comerciales, hipermercados, centros outlet) y de ocio (cines multisala, restaurantes fast food) colonizaron los polígonos $\mathrm{y}$, en algunos puntos, crearon nuevas centralidades urbanas y periurbanas. En la periferia de Girona destacan Espai Gironès, un centro comercial de $40.000 \mathrm{~m}^{2}$, derivado de una gran operación a través de dos planes parciales (Subsector 1 y 2 del Pla de Salt, 20 ha), y el área del Aeropuerto Girona-Costa Brava, que, con la expansión del modelo de vuelos de bajo coste, se ha convertido en el segundo aeropuerto de Barcelona, generando la construcción de una gran plataforma logística y terciaria en su entorno, la Central Integrada de Mercancías La Selva (plan parcial CIM la Selva, 23 ha). Por último, un fenómeno paralelo fue la proliferación del strip o carretera-mercado (Venturi, Izenour y Scott Brown, 1977; Boeri y Lanzani, 1992), esto es, la agregación espontánea a lo largo de las vías de comunicación 
de actividades de servicios (concesionarios de coches, tiendas de muebles, hoteles, etc.), debido al otorgamiento indiscriminado de licencias para edificar en suelo rústico y a la concatenación de planes parciales sin solución de coordinación municipal.

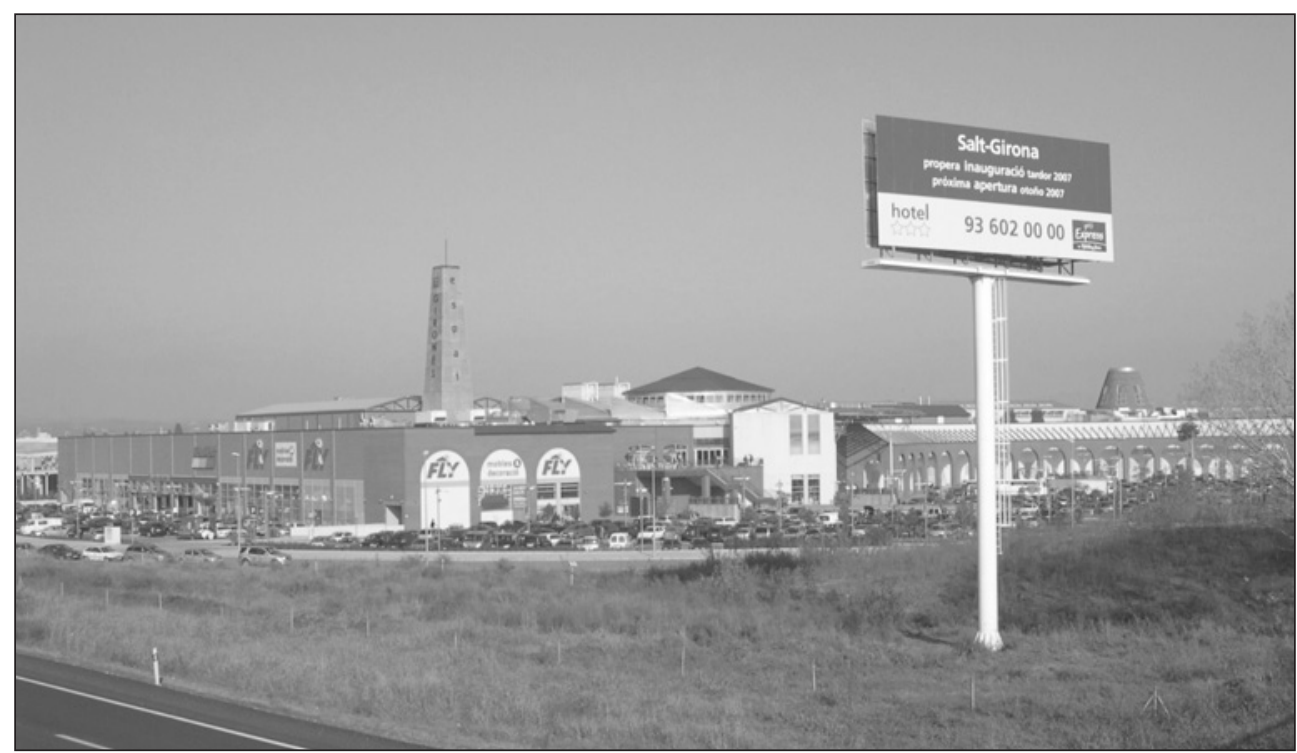

Fuente: Elaboración propia.

Muchos de estos nuevos espacios de actividad económica se han convertido en referentes locales del urbanismo global en tres sentidos básicos. En primer lugar, por el carácter transnacional de sus promotores y las empresas en ellos ubicados (Mercadona, Lidl, Decathlon, etc.). En segundo lugar, por su localización, vinculada a la nueva geografía de las redes viarias y los nudos periurbanos. En tercer lugar, por las soluciones arquitectónicas adoptadas: edificios modulares, replicables a bajo coste y de estética tematizada, funcionales para una nueva fase de la economía de cambios muy acelerados. Se trata de un paisaje líquido y de fisicidad flexible en el que los valores culturales y estéticos forjados en el propio territorio tienden a sucumbir a los estándares globalmente homologados (ver figura 8).

\section{CONCLUSIONES}

El caso de la región de Girona es un ejemplo significativo de las grandes transformaciones en el paisaje urbano-territorial que han tenido lugar en Cataluña y en España en la transición del desarrollismo a la globalización. En particular, hay que destacar la magnitud y la intensidad del proceso: entre 1979 y 2006 se aprobaron 522 planes parciales y se urbanizaron casi 6.000 ha. En el territorio esto se manifestó de manera dual. Las dinámicas de extensión 
de las ciudades a partir de ensanches compactos, con usos mixtos y densidades equilibradas, fue en paralelo a la aparición de nuevas extensiones suburbanas, especializadas y desagregadas, en los municipios de sus alrededores y los territorios en red (ensanches de baja densidad, urbanizaciones, polígonos de actividad económica). En la costa y la segunda línea de costa, la expansión estuvo marcada por la hiperespecialización en la segunda residencia a partir de proyectos, en muchos casos, dispersos o fuera de escala.

Así pues, el balance urbanístico de esta etapa es ambivalente. Por una parte, se impuso una nueva praxis urbanística ajustada a la ley, se corrigieron las patologías heredadas del desarrollismo y se obtuvo suelo para espacios públicos y equipamientos. Por otra parte, el modelo urbanístico fue alterado en tres sentidos básicos. En primer lugar, por la inercia de las prexistencias físicas y jurídicas de la etapa anterior. En segundo lugar, por la concepción sobredimensionada de muchos planes de urbanismo municipales. En tercer lugar, y último, por la modificación puntual de los planes de urbanismo de acuerdo con las demandas de oportunidad de los promotores. Así pues, a esta nueva fase de liberalización de los mercados, aceleración del giro económico y difusión urbana, le correspondieron una nueva hornada de planes de expansión y una flexibilización en su gestión para dar cabida a operaciones just in time. La dispersión apareció así como el resultado de las prácticas de free riding territorial de los promotores, aunque con el beneplácito de las administraciones y el apoyo tácito de parte de la sociedad local. La explicación radica tanto en la gestión de muchos de los ayuntamientos, atrapados en el «urbanismo» como herramienta de desarrollo como en la renuncia implícita por parte del gobierno catalán de formalizar unos criterios de planificación territorial. Su impacto ha sido una degradación del modelo territorial mediterráneo, desdibujando un sistema de núcleos forjado en coherencia ecológica y cultural con su entorno geográfico y promoviendo la aparición de paisajes banalizados, a menudo, según cánones globalmente homologados.

Ésta fue la cultura urbanística vigente hasta la aprobación del nuevo Programa de Planeamiento Territorial y, con éste, el desplegamiento de un conjunto de planes territoriales y urbanísticos supramunicipales con cuotas de crecimiento, usos e intensidades que los planes de urbanismo municipales deben observar según un principio de jerarquía normativa. A finales de 2007 estalló una crisis global que puso fin a esta etapa de onda larga y que nos invita a reflexionar profundamente sobre nuestro modelo territorial.

\section{BIBLIOGRAFÍA}

AMENDOLA, G. (2000): La ciudad postmoderna. Madrid. Celeste.

BELLET, C. (2007): «Los nuevos espacios residenciales. Estructura y paisaje», en Espacios públicos, espacios privados. Un debate sobre el territorio (Delgado, C. et al., Coords.). Asociación de Geógrafos Españoles, Departamento de Geografía, Urbanismo y Ordenación de Territorio de la Universidad de Cantabria y Gobierno de Cantabria, 93-130.

BOERI, S. y LANZANI, A. (1992): «Gli orizzonti della città diffusa», Casabella, nº 588, 44-58. BURRIEL, E. (2008): «La década prodigiosa del urbanismo español (1997-2006)», Scripta Nova, vol. XII, no 270, 26 - 30 de mayo de 2008. Universidad de Barcelona. Disponible en http://www.ub.es/geocrit/sn/sn-270/sn-270-64.htm

BURRIEL, E. (2011): «Subversion of land-use plans and the housing bubble in Spain», Urban Research \& Practice, $\mathrm{n}^{\circ}$ 4:3, 232-249. 
CASTAÑER, M.; GUTIÉRREZ, O.; VICENTE, J. (2009): «Mobilitat laboral, àrees de cohesió i àrees de planificació a Catalunya», Treballs de la Societat Catalana de Geografia, $n^{\circ}$ 67-68, 61-86.

DONAIRE, J.A. (1996): El turismo a los ojos del posmodernismo. Una lectura desde la dialéctica socioespacial. La Costa Brava, Tunicia y los malls. Tesis de doctorado, Bellaterra, Universidad Autónoma de Barcelona.

ELLIN, N. (1999): Postmodern urbanism. Nueva York. Princeton Architectural Press.

ESTEBAN, J. (2003): «Perspectivas de la ordenación territorial», en Planeamiento urbanístico. De la controversia a la renovación (Font, A., Coord.). Barcelona, Diputació de Barcelona, 207-219.

FERRER, A. y SABATÉ, J. (1999): «L'urbanisme municipal», en 20 Anys d'ajuntaments democràtics (Nel·lo, O., Dir.). Barcelona, Federació de Municipis de Catalunya, 117-159.

FRAGUELL, R.M. (1994): Turisme residencial i territori: la segona residència a la Regió de Girona. Girona. l'Eix editorial.

FRANQUESA, J. (2010): Les comunitats jardí a Catalunya. Una experiència urbana retrobada. Barcelona. Icaria.

GOSPODINI, A. (2006): «Portraying, classifying and understanding the emerging landscapes in the post-industrial city», Cities, $\mathrm{n}^{\circ} 23(5), 311-330$.

INDOVINA, F. (1990): La città diffusa. Venecia. IUAV- DAEST.

MONCLÚS, F.J. (1998): La ciudad dispersa. Barcelona. Centre de Cultura Contemporània de Barcelona.

MUÑIZ, I.; CALATAYUD, D. y GARCIA, M. Á. (2007): «Sprawl. Causas y consecuencias de la dispersión urbana», en La ciudad de baja densidad. Lógicas, gestión y contención (Indovina, F., ed), Barcelona, Diputación de Barcelona, 307-347.

MUÑOZ, F. (2004): UrBANALització. La producció residencial de baixa densitat a la província de Barcelona, 1985-2001. Tesis de doctorado, Bellaterra, Universidad Autónoma de Barcelona.

NAREDO, J.M. y MONTIEL, A. (2011): El modelo inmobiliario español y su culminación en el caso valenciano. Barcelona. Icaria.

NEL·LO, O. (2001): Ciutat de ciutats. Reflexions sobre el procés d'urbanització a Catalunya. Barcelona. Empúries (trad. castellano: Cataluña, ciudad de ciudades. Lleida. Milenio).

NEL·LO, O. (2003): Aquí, no! Els conflictes territorials a Catalunya. Barcelona. Empúries.

NEL·LO, O. (2011): «El planeamiento territorial en Cataluña», Cuadernos Geográfi$\cos , \mathrm{n}^{\circ} 47$ (2010-2), 131-167. Disponible en http://www.ugr.es/ cuadgeo/docs/articulos/047/047-006.pdf

NOGUÉ, J. (2007): «Territorios sin discurso, paisajes sin imaginario: retos y dilemas», Ería, $\mathrm{n}^{\circ} 73-74,373-382$.

ROMERO, J. (2010): Geopolítica y gobierno del territorio en España. Valencia. Tirant lo Blanch.

ROMERO, J. (2011): «Construcción residencial y gobierno del territorio en España. De la burbuja especulativa a la recesión. Causas y consecuencias», Cuadernos Geográfi$\cos , \mathrm{n}^{\circ} 47$ (2010-2), 131-167. Disponible en http://www.ugr.es/ cuadgeo/docs/articulos/047/047-002.pdf 
RUEDA, S. (2002): «Els costos ambientals dels models urbans dispersos», Papers. Regió Metropolitana de Barcelona, $\mathrm{n}^{\circ}$ 36, 73-104.

TERRITORI - OBSERVATORI DE PROJECTES I DEBATS TERRITORIALS DE CATALUNYA: http://territori.scot.cat/ (consulta: Marzo de 2012)

VALDUNCIEL, J. (2011): Paisatge i models urbans contemporanis. Les Comarques Gironines (1979-2006): del desarrollismo a la globalització. Tesis de doctorado, Girona, Universidad de Girona.

VENTURI, R.; IZENOUR, S.; SCOTT BROWN, D. (1977): Learning from Las Vegas. The forgotten symbolism of architectural form. Cambridge. MIT Press.

VICENTE, J. (1998): El procés de construcció de l'àrea urbana de Girona. Plans, discursos i realitats. Tesis de doctorado, Bellaterra, Universitat Autònoma de Barcelona.

VICENTE, J. (2003): «La Regió de Girona: un model territorial en transformació, amb potencial i fràgil», Papers. Regió Metropolitana de Barcelona, 39, 61-72.

VVAA (2006): Los procesos urbanos postfordistas. Actas del VIII Coloquio y Jornadas de Campo de Geografía Urbana. Editado por A. Artigues, A. Bauzá, M. Blázquez, J.M. González, I. Murria y O. Rullán, Universitat de les Illes Balears y Asociación de Geógrafos Españoles, Palma, 2007. 